\title{
Effect of Inactivation of $\operatorname{deg} S$ on Salmonella enterica Serovar Typhimurium In Vitro and In Vivo
}

\author{
Gary Rowley, ${ }^{1}$ Andrew Stevenson, ${ }^{1}$ Jan Kormanec, ${ }^{2}$ and Mark Roberts ${ }^{1 *}$ \\ Molecular Bacteriology Group, Institute of Comparative Medicine, Department of Veterinary Pathology, \\ Glasgow University Veterinary School, Glasgow, United Kingdom, ${ }^{1}$ and Institute of Molecular \\ Biology, Centre of Excellence for Molecular Medicine, Slovak Academy \\ of Science, Dubravska, Bratislava, Slovak Republic ${ }^{2}$
}

Received 4 June 2004/Returned for modification 8 July 2004/Accepted 17 September 2004

\begin{abstract}
The alternative sigma factor $\left(\operatorname{RpoE} \sigma^{\mathbf{E}}\right)$ enables Salmonella enterica serovar Typhimurium to adapt to stressful conditions, such as oxidative stress, nutrient deprivation, and growth in mammalian tissues. Infection of mice by Salmonella serovar Typhimurium also requires $\sigma^{\mathrm{E}}$. In Escherichia coli, activation of the $\sigma^{\mathrm{E}}$ pathway is dependent on proteolysis of the anti-sigma factor RseA and is initiated by DegS. DegS is also important in order for $E$. coli to cause extraintestinal infection in mice. We constructed a $\operatorname{deg} S$ mutant of the serovar Typhimurium strain SL1344 and compared its behavior in vitro and in vivo with those of its wild-type (WT) parent and an isogenic rpoE mutant. Unlike $E$. coli $\operatorname{deg} S$ strains, the Salmonella serovar Typhimurium $\operatorname{deg} S$ strain grew as well as the WT strain at $42^{\circ} \mathrm{C}$. The $\operatorname{deg} S$ mutant survived very poorly in murine macrophages in vitro and was highly attenuated compared with the WT strain for both the oral and parenteral routes of infection in mice. However, the $\operatorname{deg} S$ mutant was not as attenuated as the serovar Typhimurium rpoE mutant: 100- to 1,000-fold more $\operatorname{deg} S$ bacteria than $r p o E$ bacteria were present in the livers and spleens of mice $24 \mathrm{~h}$ after intraperitoneal challenge. In most assays, the $r p o E$ mutant was more severely affected than the $\operatorname{deg} S$ mutant and a $\sigma^{\mathrm{E}}$-dependent reporter gene was more active in the $\operatorname{deg} S$ mutant than the $r p o E$ strain. These findings indicate that $\operatorname{deg} S$ is important for activation of the $\sigma^{\mathrm{E}}$ pathway in serovar Typhimurium but that alternative pathways for $\sigma^{\mathrm{E}}$ activation probably exist.
\end{abstract}

In order for bacteria to survive under a wide range of environmental pressures, including survival in both warm- and cold-blooded hosts, they have evolved a variety of stress responses. A number of the stress responses involve alternative sigma factors, which change the promoter specificity of RNA polymerase. We have previously reported that in Salmonella enterica serovar Typhimurium, one of the alternative sigma factors, $\sigma^{\mathrm{E}}$, encoded by $r p o E$, is critical for virulence in a mouse model (13). Salmonella serovar Typhimurium $\sigma^{\mathrm{E}}$ is also required for defense against oxidative stress and antimicrobial peptides and for survival within macrophages and in stationary phase $(13,25)$. Unlike their Escherichia coli counterparts, Salmonella serovar Typhimurium rpoE mutants are able to grow normally at $43^{\circ} \mathrm{C}(13)$. Also, in laboratory strains of $E$. coli, $r p o E$ is necessary for growth at $37^{\circ} \mathrm{C}$, but this is not the case for Salmonella serovar Typhimurium and other bacteria $(6,8,10$, 11, 13, 17, 25, 27).

Regulation of the $\sigma^{\mathrm{E}}$ pathway has been studied extensively with $E$. coli laboratory strains. Under nonstress conditions, $\sigma^{\mathrm{E}}$ is inactive due to its sequestration to the cytoplasmic face of the inner membrane by its cognate anti-sigma factor, RseA (1). Liberation of $\sigma^{\mathrm{E}}$ from RseA and activation of the $\sigma^{\mathrm{E}}$ pathway is dependent on a two-stage cleavage of RseA by two proteases located in the inner membrane, DegS and YaeL (EcfE) (1). DegS possesses a membrane anchor, a serine protease domain,

\footnotetext{
* Corresponding author. Mailing address: Institute of Comparative Medicine, Professor of Molecular Bacteriology, Department of Veterinary Pathology, Glasgow University Veterinary School, Bearsden Road, Glasgow G61 1QH, United Kingdom. Phone: 01413305780. Fax: 0141330 5602. E-mail: m.roberts@vet.gla.ac.uk.
}

and a PDZ domain (3). In the absence of stress, the DegS PDZ domain inhibits its protease domain. The PDZ domain recognizes a YQF peptide sequence at the $C$ termini of outer membrane porins that accumulate in the periplasm due to envelope stress or artificially, due to overexpression of porins (26). This activates DegS protease activity, leading to cleavage of the periplasmic domain of RseA (26). YaeL, the second protease involved in RpoE activation, acts on the cytoplasmic domain of RseA to liberate $\sigma^{\mathrm{E}}$. Like that of DegS, the activity of YaeL is also negatively controlled by its PDZ domain $(5,16)$. In E. coli, YaeL is not capable of cleaving RseA and liberating RpoE from RseA in the absence of DegS $(2,15)$.

For laboratory strains of $E$. coli, $\operatorname{deg} S$ has been reported to be an essential gene, but the importance of DegS is dependent on the background of the strains (3). More recently, a $\operatorname{deg} S$ mutant of a wild-type (WT) virulent extraintestinal strain of $E$. coli has been reported (20). This strain lacks a number of phenotypes associated with $\operatorname{deg} S$ mutants of $E$. coli laboratory strains, and the mutant was fully viable (20). However, the $E$. coli $\operatorname{deg} S$ mutant was attenuated in murine systemic and urinary tract infection models, although it is not known if this was due to the $\operatorname{deg} S$ mutation affecting activation of the $\sigma^{\mathrm{E}}$ regulon (20).

Here we report the construction of a Salmonella serovar Typhimurium $\operatorname{deg} S$ mutant and its characterization in vitro and in vivo.

\section{MATERIALS AND METHODS}

Bacterial strains, plasmids, and growth conditions. Bacterial strains and plasmids used or constructed in this study are listed in Table 1. All strains were cultivated in Luria-Bertani (LB) broth or on LB agar (LA) plates (22). When 
TABLE 1. Bacterial strains and plasmids used in this study

\begin{tabular}{|c|c|c|}
\hline Strain or plasmid & Genotype or relevant characteristics & $\begin{array}{l}\text { Source or } \\
\text { reference }\end{array}$ \\
\hline \multicolumn{3}{|c|}{ Serovar Typhimurium strains } \\
\hline SL1344 & his $G$; virulent mouse strain; $\mathrm{Sm}^{\mathrm{r}}$ & 12 \\
\hline 12023 & Virulent mouse strain & 21 \\
\hline GVB311 & SL1344 rpoE::Km ${ }^{\mathrm{r}}$ & 13 \\
\hline GVB1340 & $12023 \Delta \operatorname{deg} S:: \mathrm{Km}^{\mathrm{r}}$ & This study \\
\hline GVB1362 & SL1344 $\Delta \operatorname{deg} S:: \mathrm{Km}^{\mathrm{r}}$ & This study \\
\hline \multicolumn{3}{|l|}{ Plasmids } \\
\hline pKD4 & pANT-S $\gamma$ derivative containing an FRT-flanked $\mathrm{Km}^{\mathrm{r}}$ gene; $\mathrm{Ap}^{\mathrm{r}}$ & 7 \\
\hline pKD46 & pINT-ts derivative containing ara $C-P_{a r a B}$ and $\gamma \beta$ exo DNA fragments; $\mathrm{Ap}^{\mathrm{r}}$ & 7 \\
\hline pTL61t & Low-copy-number, promotorless lac $Z$ fusion vector; $A p^{\mathrm{r}}$ & 18 \\
\hline prpoEP3 & pTL61t with HindIII/XbaI-cloned RpoE-dependent rpoE promoter; Ap ${ }^{\mathrm{r}}$ & 19 \\
\hline
\end{tabular}

required, media were supplemented with $100 \mu \mathrm{g}$ of ampicillin (Sigma)/ml, $75 \mu \mathrm{g}$ of kanamycin (Invitrogen)/ml, or $100 \mu \mathrm{g}$ of streptomycin (Sigma)/ml. For analysis of growth kinetics, strains were grown overnight in the appropriate medium. Overnight cultures were then diluted 100 -fold into $50-\mathrm{ml}$ aliquots of the same medium and incubated at the desired temperature, and growth was monitored spectrophotometrically.

Construction of a $\operatorname{deg} S$ mutant. A $\operatorname{deg} S$ mutant was constructed by using the $\lambda$ Red mutagenesis system (7). We designed mutagenesis primers degSRedF (5' TCCATCATGTTTGTGAAGCTCTTACGTTCGGTCG CAATAGGTTTAAT

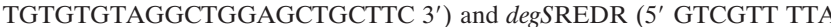
GTTCGACGCCGGGTATTCCTGCACCGTCACCTGGAACGTGACATAT GAATATCCTCCTTAG $3^{\prime}$ ) to amplify the kanamycin cassette from plasmid pKD4, with homologous flanking for the start and end of the degS coding sequence. This ensures complete deletion of the gene as well as inserting a kanamycin gene marker. Initially mutagenesis was carried out in serovar Typhimurium 12023, because we find $\lambda$ Red mutagenesis is more efficient in this background. After mutagenesis, colonies were recovered on LA plates containing kanamycin. Mutagenesis was confirmed by PCR using primers degSEXTF ( $5^{\prime}$ GCGGCAACGAGAACATTTAT $3^{\prime}$ ) and degSEXTR (5' CCAGTCTTTATTG ACTAGTC $3^{\prime}$ ), which are external to the site of mutagenesis, as well as primers that amplify the kanamycin cassette in order to confirm the orientation of the marker (7). After confirmation, the mutation was moved by P22 transduction into serovar Typhimurium strain SL1344 and again confirmed by PCR.

Infection of murine macrophages. The ability of serovar Typhimurium strains to invade, and to survive and replicate within, the murine macrophage cell line RAW 264.7 was assayed essentially as previously described $(14,24)$. Briefly, a multiplicity of infection of $\sim 1: 1$ was used, and the number of bacteria inside infected cells was determined at 3 and $24 \mathrm{~h}$ postinfection by a gentamicin protection assay $(9,24)$. Each assay was repeated three times, and statistical significance was measured by using analysis of variance (ANOVA).

Analysis of virulence. For all in vivo studies, strains were grown statically overnight at $37^{\circ} \mathrm{C}$, centrifuged, washed, resuspended to the appropriate concentration in sterile phosphate-buffered saline, and administered to mice in doses of $200 \mu \mathrm{l}$. Female BALB/c mice ( 6 to 8 weeks old; Harlan UK) were used throughout. Initial assessment of virulence was carried out by using the well-described competition index assay $(4,14)$.

For oral infection, the inoculum was administered via oral gavage, and mice were culled 7 days later. Organs (livers, spleens, Peyer's patches, and mesenteric lymph nodes) were isolated and homogenized, and numbers of bacteria present were determined by viable counting. Statistical significance was analyzed by ANOVA.

$\boldsymbol{\beta}$-Galactosidase assay. Overnight cultures of strains harboring the prpoEP3 reporter plasmid (19) were diluted 1:100 into fresh LB broth with appropriate antibiotics and then incubated at $37^{\circ} \mathrm{C}$ with aeration until they reached stationary phase. $\beta$-Galactosidase activities of the cultures were assayed in 96-well plates (23). A mean activity was calculated from seven samples. Statistical significance was measured by using a two-tailed Student $t$ test, with Welch's correction, and a $P$ value of $<0.05$ was considered significant.

\section{RESULTS}

Construction of a serovar Typhimurium $\operatorname{deg} S$ mutant. Because $\sigma^{\mathrm{E}}$ is not essential for serovar Typhimurium viability, we predicted that $\operatorname{deg} S$ could also be successfully mutated in an otherwise WT background, unless DegS was necessary to perform another function unrelated to $\sigma^{\mathrm{E}}$ activation. Using $\lambda$ Red mutagenesis (7), we replaced the coding sequence of serovar Typhimurium SL1344 $\operatorname{deg} S$ with a kanamycin resistance cassette as described above to generate strain GVB1362. The colony morphology of the SL1344 $\operatorname{deg} S$ mutant was not different from that of the WT strain.

Effect of the $\operatorname{deg} S$ mutation on $\sigma^{\mathrm{E}}$ activity in serovar Typhimurium. We have previously shown that serovar Typhimurium rpoE is regulated by three promoters, with the third promoter, rpoEP3, autoregulated by RpoE itself (19). Expression of an rpoEP3::lacZ reporter gene increases in WT serovar Typhimurium in late-logarithmic phase. We compared the expression of the rpoEP3::lacZ gene in WT SL1344 and the isogenic $\operatorname{deg} S$ and $r p o E$ mutants after $6 \mathrm{~h}$ of growth in LB broth (Fig. $1 \mathrm{~A})$. Interestingly, in the $\operatorname{deg} S$ mutant, the activity of the rpoEP3 promoter is twice that of the rpoE strain $(P<0.05)$. This indicates the presence of free $\sigma^{\mathrm{E}}$ within the $\operatorname{deg} S$ mutant, although the activity of the reporter gene is much lower than that in the WT strain (Fig. 1A). The background $\beta$-galactosidase activity measured in the rpoE strain is consistent with that of the empty vector (19).

The $\operatorname{deg} S$ mutation affects the growth rate of serovar Typhimurium. A serovar Typhimurium rpoE mutant exhibits aberrant growth under certain conditions (13). In particular, a serovar Typhimurium rpoE mutant exhibits an extended lag phase and reaches a lower final optical density (OD) when grown aerobically in LB broth (13). Theoretically, and on the basis of evidence derived from E. coli $\operatorname{deg} S$ studies, a $\operatorname{deg} S$ mutant should possess phenotypic characteristics similar to those previously observed for the rpoE mutant. Therefore, we compared the growth of the $\operatorname{deg} S$ mutant with that of the rpoE mutant and the WT strain in shaking LB-broth cultures at $37^{\circ} \mathrm{C}$ (Fig. 1B). The $\operatorname{deg} S$ strain had a longer lag phase than the WT strain, though not as protracted as that of the rpoE mutant. The final ODs of the $\operatorname{deg} S$ and WT strains were very similar. In contrast, the rpoE mutant entered stationary phase prematurely, as previously observed (13).

A $\operatorname{deg} S$ mutant of a WT extraintestinal isolate of $E$. coli was unable to grow in LB broth containing 3\% ethanol (20). The WT E. coli strain was able to grow under the same conditions, although at a lower growth rate than in LB broth alone (20). We examined the ability of serovar Typhimurium strains to 
A
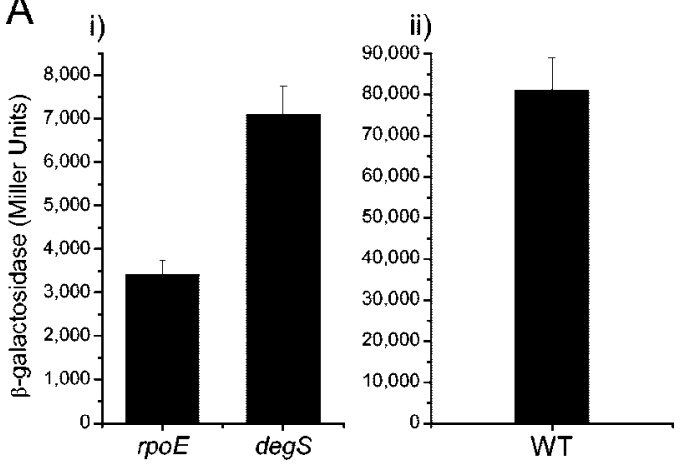

B
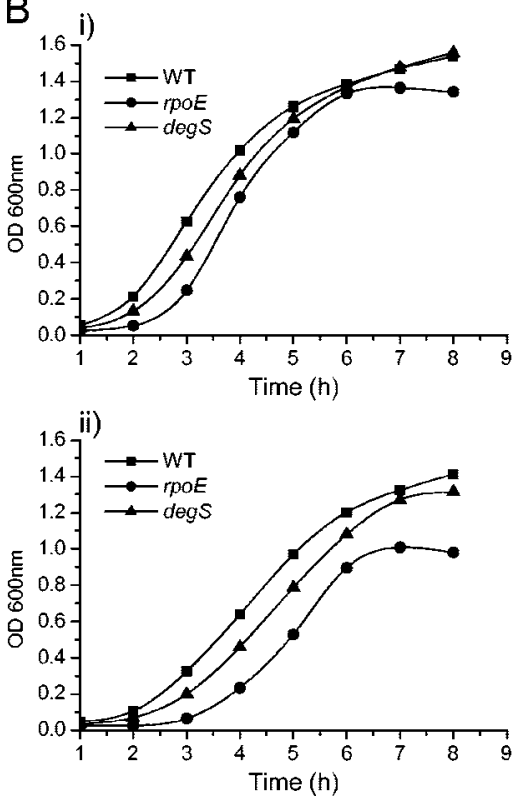

FIG. 1. Effects of inactivation of $\operatorname{deg} S$ on activity of the $\sigma^{\mathrm{E}}$ pathway and growth of Salmonella serovar Typhimurium. (A) Activity of the $\sigma^{\mathrm{E}}$ pathway measured by using the $\sigma^{\mathrm{E}}$-dependent prpoEP3::lac $Z$ reporter gene. $\beta$-Galactosidase activities were determined for the $r p o E, \operatorname{deg} S$ (i), and WT (ii) serovar Typhimurium strains harboring the prpoEP3 reporter plasmid. Strains were grown for $6 \mathrm{~h}$ (late-log phase) in LB broth at $37^{\circ} \mathrm{C}$ with aeration. Bars represent means of seven replicates; error bars, standard deviations of the means. (B) Effects of the $\operatorname{deg} S$ and rpoE mutations on aerobic growth of serovar Typhimurium at $37^{\circ} \mathrm{C}$ in LB broth (i) and in LB broth plus 3\% ethanol (ii). Growth was monitored for $8 \mathrm{~h}$ and measured spectrophotometrically $\left(\mathrm{OD}_{600}\right)$.

grow in LB broth in the presence of 3\% ethanol (Fig. 1B). As with $E$. coli, ethanol reduced the growth rate of the WT strain relative to that in LB broth alone. However, unlike the E. coli mutant, the serovar Typhimurium $\operatorname{deg} S$ mutant was able to grow in $3 \%$ ethanol, although slightly less well than the WT strain; the difference between the strains was very similar to that seen in LB broth alone. The rpoE mutant was also able to grow in LB broth plus 3\% ethanol, but its growth was more severely affected than that of the other two strains. The rpoE mutant exhibited a longer lag phase than the other strains, and its exponential phase was shorter. The results show that $3 \%$ ethanol is a stressful environment for serovar Typhimurium

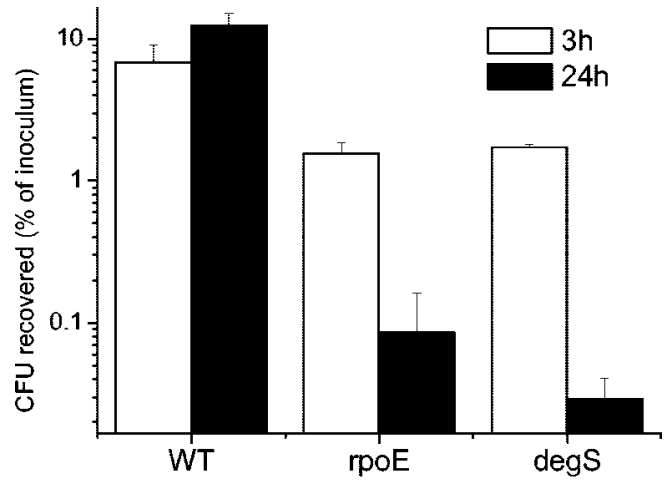

FIG. 2. Effects of the $\operatorname{degS}$ mutation on Salmonella serovar Typhimurium invasion and survival in macrophages. Bacteria at a multiplicity of infection of $\sim 1: 1$ were incubated with the murine macrophage cell line RAW 264.7. The assay was performed as described in the text. Graphs show the number of viable bacteria (as a percentage of the initial inoculum) inside the macrophage at $3 \mathrm{~h}$ (white bars) and $24 \mathrm{~h}$ (black bars) after infection. Each bar represents the mean from triplicate experiments; error bars, standard deviations.

but that the ability of the $\operatorname{deg} S$ mutant to survive and replicate in this environment is greater than that of the $r p o E$ mutant.

The ability of a serovar Typhimurium $\operatorname{deg} S$ mutant to invade and survive within a murine macrophage cell line is similar to that of a serovar Typhimurium rpoE mutant. Given that a serovar Typhimurium rpoE mutant survives poorly in murine macrophages, we hypothesized that the intracellular environment would also be harsh for a serovar Typhimurium $\operatorname{deg} S$ mutant. At $3 \mathrm{~h}$ after infection, significantly lower numbers $(P<$ 0.05 ) of the rpoE and $\operatorname{deg} S$ mutants than of the WT strain were present inside RAW 264.7 cells (Fig. 2). The WT strain replicated inside RAW 264.7 cells between 3 and $24 \mathrm{~h}$ postinfection; in contrast, during the same period, the intracellular numbers of the $\operatorname{deg} S$ and $r p o E$ mutants decreased to less than $1 / 10$ of their initial numbers. This indicates that the $\operatorname{deg} S$ and $r p o E$ mutants (as previously shown) are killed within macrophages. There was no significant difference $(P<0.05)$ between the intracellular numbers of the $r p o E$ and $\operatorname{deg} S$ mutants at 3 or $24 \mathrm{~h}$.

The serovar Typhimurium $\operatorname{deg} S$ mutant is severely attenuated in a mouse infection model but is not as attenuated as a serovar Typhimurium rpoE mutant. $\sigma^{\mathrm{E}}$ is essential for serovar Typhimurium to cause infection of mice by either the oral or the parenteral route of infection (13). We would expect, therefore, that $\operatorname{deg} S$ would play a similarly important role in serovar Typhimurium virulence during both the systemic and oral phases of infection of mice.

We initially compared the virulence of the WT and $\operatorname{deg} S$ strains by a competition assay in which $\sim 1 \times 10^{3} \mathrm{CFU}$ of both the WT and $\operatorname{deg} S$ strains was given to mice in a mixed infection via the intraperitoneal (i.p.) route. A competitive index (CI) of $\sim 1$ indicates that the strains are of equal virulence. The CI for $\operatorname{deg} S$ versus the WT is 0.006 , indicating that the $\operatorname{deg} S$ mutant is severely attenuated. When a similar experiment was performed with the rpoE mutant versus the WT, the CI could not be determined accurately, because no CFU of the rpoE mutant could be isolated from either the liver or the spleen (data not shown). 
A

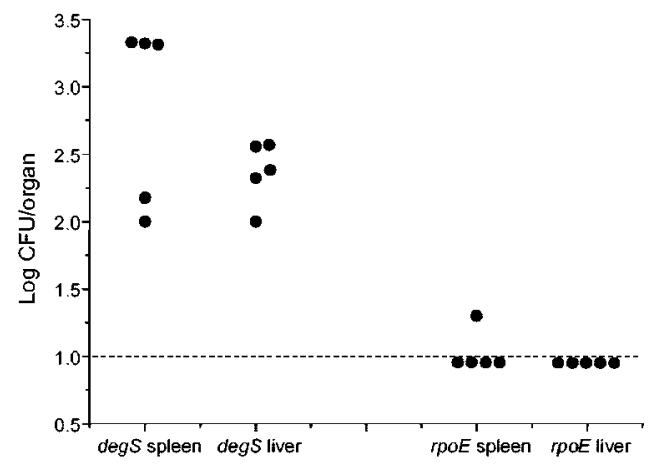

B

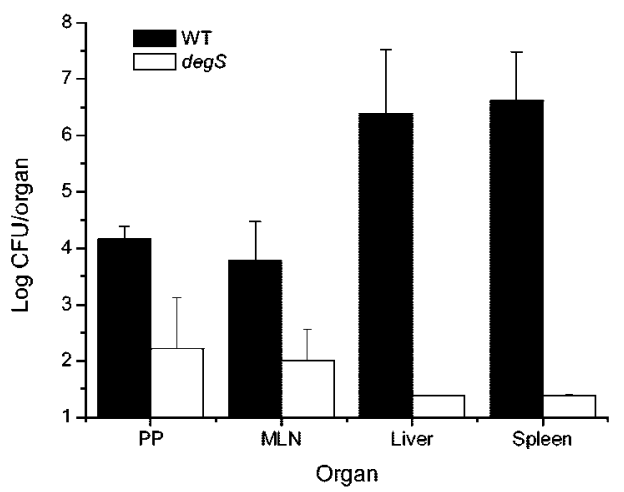

FIG. 3. Effect of inactivation of $\operatorname{deg} S$ on the ability of Salmonella serovar Typhimurium to infect mice. (A) Comparison of the abilities of serovar Typhimurium $\operatorname{deg} S$ and $r p o E$ mutants to survive in vivo in murine organs following parenteral administration. Groups of five female BALB/c mice were challenged i.p. with $10^{5} \mathrm{CFU}$ of either the rpoE or the $\operatorname{degS}$ mutant. After $24 \mathrm{~h}$, bacteria in the spleens and livers were enumerated. Each filled circle represents the count from the organ of an individual mouse. (B) Effect of the $\operatorname{deg} S$ mutation on the ability of serovar Typhimurium to infect mice via the oral route. Mice were challenged orally with either $5 \times 10^{5} \mathrm{CFU}$ of the WT strain or 1.6 $\times 10^{8} \mathrm{CFU}$ of the $\operatorname{deg} S$ mutant, and the numbers of bacteria present in different organs were determined 7 days later. Each bar represents the mean for four mice; error bars, standard deviations. The error bars for livers and spleens of the $\operatorname{deg} S$ group are too small to be visible. PP, Peyer's patches; MLN, mesenteric lymph nodes.

The $r p o E$ and $\operatorname{deg} S$ strains possess the same selectable marker $\left(\mathrm{Km}^{\mathrm{r}}\right)$; therefore, it is not possible to compare the virulence of the rpoE and $\operatorname{deg} S$ mutants by the standard competition assay. Instead, we gave single doses of $10^{5} \mathrm{CFU}$ of the $r p o E$ or $\operatorname{deg} S$ mutant individually via the i.p. route to groups of five mice and enumerated the bacteria of each strain present in the spleens and livers of individual mice $24 \mathrm{~h}$ later (Fig. 3A). Bacteria could be isolated from the spleens and livers of all of the mice infected with the $\operatorname{deg} S$ mutant. The numbers of $\operatorname{deg} S$ bacteria isolated ranged from $\sim 100$ to 2,000 CFU. In contrast, the $r p o E$ mutant was isolated from only one of the mice and from only one organ (the spleen). All of the parenteral infection studies indicate that the $\operatorname{deg} S$ mutant is less severely attenuated than the rpoE mutant.

We also investigated the role of DegS during infection via the oral route. Mice were inoculated orally with either $5 \times 10^{5}$
CFU of the WT strain or $1.6 \times 10^{8} \mathrm{CFU}$ of the $\operatorname{degS}$ mutant, and the numbers of bacteria present were determined 7 days later (Fig. 3B). Even though the dose of the $\operatorname{degS}$ mutant was much higher than that of the WT strain, there were significantly fewer $\operatorname{deg} S$ than WT bacteria in all organs examined $(P$ $<0.05)$. The most dramatic difference can be seen in the liver and spleen, where the numbers of WT bacteria isolated were $\sim 10^{6}$-fold greater than those for the $\operatorname{deg} S$ strain.

\section{DISCUSSION}

We have previously shown that Salmonella serovar Typhimurium $\sigma^{\mathrm{E}}$ is an important regulator of defense against a variety of stresses and is critically important for survival of serovar Typhimurium in vivo. The majority of the research on the activation and regulation of the $\sigma^{\mathrm{E}}$ pathway has been carried out on laboratory strains of $E$. coli. The number of genes and the organization of the rpoE operons of E. coli and Salmonella serovar Typhimurium are identical (13).

As with $r p o E$, $\operatorname{degS}$ does not appear to be essential for serovar Typhimurium under standard growth conditions in vitro. The colonies of a $\operatorname{deg} S$ mutant of a clinical $E$. coli strain were more translucent than those of the parenteral strain (20). The colony morphology of the Salmonella serovar Typhimurium $\operatorname{deg} S$ strain was identical to that of its WT parent on LA plates (data not shown). At $37^{\circ} \mathrm{C}$ in $\mathrm{LB}$ broth, the $\operatorname{degS}$ mutant demonstrated an extended lag phase in comparison to the WT strain, but this lag phase was not as protracted as that of the $r p o E$ mutant. The growth patterns of the three strains were the same at $43^{\circ} \mathrm{C}$ (data not shown). In the presence of $3 \%$ ethanol, we found that the WT, rpoE, and $\operatorname{deg} S$ strains all had reduced growth rates but displayed similar kinetics in relation to each other as they did at $37^{\circ} \mathrm{C}$, the only difference being that the $r p o E$ mutant entered stationary phase at a lower OD at 600 $\mathrm{nm}\left(\mathrm{OD}_{600}\right)$ than the other two strains. Thus, in terms of growth, the $\operatorname{deg} S$ mutant of serovar Typhimurium does not behave identically to an rpoE mutant. The effects of inactivation of $\operatorname{degS}$ on growth at elevated temperatures and in the presence of ethanol are much smaller for Salmonella serovar Typhimurium than for a clinical strain of E. coli (20).

The difference between the growth kinetics of the $\operatorname{deg} S$ and rpoE serovar Typhimurium strains suggests that there may be active (free) $\sigma^{\mathrm{E}}$ present in the $\operatorname{deg} S$ mutant strain. This is supported by the finding that the rpoEP3::lac $Z$ reporter gene is $\sim 2$-fold more active in the $\operatorname{deg} S$ mutant than in the $r p o E$ mutant.

The $\operatorname{deg} S$ mutation significantly reduced the ability of serovar Typhimurium to replicate and/or survive intracellularly within macrophages. However, there was no significant difference between the intracellular behaviors of the $\operatorname{deg} S$ and $r p o E$ mutants. This was the only assay in which a difference between the serovar Typhimurium rpoE and $\operatorname{deg} S$ mutants was not found. There may be a number of reasons for this. It is possible that the resolving power of the assay is not sufficient to differentiate between the two mutants. Alternatively, if there is a $\operatorname{deg} S$-independent pathway for activation of the $\sigma^{\mathrm{E}}$ pathway (see below), it may not be activated by the intracellular environment of RAW 264.7 cells in vitro.

The serovar Typhimurium rpoE mutant used in this study (GVB311) is severely attenuated in a mouse model via both 
the oral and parenteral infection routes, and when used as a live vaccine strain, it provides no protection against WT serovar Typhimurium (13). The $\operatorname{degS}$ mutant is also severely attenuated, although less so than the $r p o E$ mutant. For example, when $\sim 1 \times 10^{5} \mathrm{CFU}$ of the rpoE or $\operatorname{deg} S$ mutant was given to mice i.p., neither strain was able to establish infection in the liver or the spleen, and both were cleared rapidly. However, whereas $\operatorname{deg} S$ bacteria could be isolated from the livers and spleens of all infected mice, the $r p o E$ mutant could be isolated only (in low numbers) from the spleen of one of five animals $24 \mathrm{~h}$ after challenge. Therefore, the $\operatorname{deg} S$ mutant survived at least 100 - to 1,000 -fold better than the rpoE mutant.

The ability of the $\operatorname{deg} S$ mutant to infect mice via the oral route was compared with that of the WT strain. Higher numbers of WT than $\operatorname{degS}$ bacteria were found in all organs at 7 days postinfection despite the fact that mice received a $\sim 300$ fold-lower dose of the WT strain. The WT strain was found in higher numbers at systemic sites (liver and spleen) than at gut-associated sites (Peyer's patches and mesenteric lymph nodes). The pattern was reversed for the $\operatorname{deg} S$ mutant, with more bacteria recovered from the Peyer's patches and mesenteric lymph nodes than from the liver and spleen. Interestingly, this is also the case for serovar Typhimurium strains with mutations in $r p o E$ and the $\sigma^{\mathrm{E}}$-regulated genes $h \operatorname{tr} A$ and $\operatorname{sur} A$ $(13,24)$. This indicates either that $\sigma^{\mathrm{E}}$-regulated genes are important for translocation of serovar Typhimurium to systemic sites from the gut-associated lymphoid tissue or that $\sigma^{\mathrm{E}}$-regulated genes are more important for survival at systemic sites, or both. The fact that $\operatorname{deg} S$ and $r p o E$ mutants are highly attenuated when administered parenterally suggests that the latter possibility is more likely.

In all of our studies, except the macrophage infection assay, inactivation of $\operatorname{deg} S$ had a less severe effect on serovar Typhimurium than inactivation of $r p o E$. This suggests that the $\sigma^{\mathrm{E}}$ pathway can be activated in a $\operatorname{deg} S$-independent manner, although not to the same extent as when DegS is present. It may be that in certain situations YaeL can cleave RseA and liberate $\sigma^{\mathrm{E}}$ in the absence of DegS. The PDZ domains of both DegS and YaeL negatively regulate their proteolytic activity against RseA (1). E. coli YaeL lacking the PDZ domain (YaeL $\Delta$ PDZ) can activate the $\sigma^{\mathrm{E}}$ pathway in the absence of $\operatorname{DegS}(5,16)$. In fact, activation of $\sigma^{\mathrm{E}}$ by YaeL $\Delta \mathrm{PDZ}$ is more efficient in a $\operatorname{deg} S$ than in a WT background (5). Therefore, in the Salmonella serovar Typhimurium $\operatorname{deg} S$ strain, signals that interact with the PDZ domain of YaeL could lead to activation of $\sigma^{\mathrm{E}}$. Interestingly, the level of free $\sigma^{\mathrm{E}}$ present within serovar Typhimurium $\operatorname{deg} S$, though low, is enough to partially correct some of the severe phenotypes observed with the serovar Typhimurium $r p o E$ mutant, including in vivo survival. We are currently investigating DegS-independent activation of the serovar Typhimurium $\sigma^{\mathrm{E}}$ regulon.

\section{ACKNOWLEDGMENTS}

This work and G.R. were supported by grant 062631 from the Wellcome Trust. J.K was partially supported by grant $2 / 3010 / 23$ from the Slovak Academy of Sciences and by Wellcome Trust grant 065027/Z/ $01 / \mathrm{Z}$.

\section{REFERENCES}

1. Alba, B. M., and C. A. Gross. 2004. Regulation of the Escherichia coli sigma-dependent envelope stress response. Mol. Microbiol. 52:613-619.

2. Alba, B. M., J. A. Leeds, C. Onufryk, C. Z. Lu, and C. A. Gross. 2002. DegS and YaeL participate sequentially in the cleavage of RseA to activate the $\sigma^{\mathrm{E}}$-dependent extracytoplasmic stress response. Genes Dev. 16:2156-2168.

3. Alba, B. M., H. J. Zhong, J. C. Pelayo, and C. A. Gross. 2001. $\operatorname{deg} S(h h o B)$ is an essential Escherichia coli gene whose indispensable function is to provide sigma activity. Mol. Microbiol. 40:1323-1333.

4. Beuzon, C. R., and D. W. Holden. 2001. Use of mixed infections with Salmonella strains to study virulence genes and their interactions in vivo. Microbes Infect. 3:1345-1352.

5. Bohn, C., J. Collier, and P. Bouloc. 2004. Dispensable PDZ domain of Escherichia coli YaeL essential protease. Mol. Microbiol. 52:427-435.

6. Craig, J. E., A. Nobbs, and N. J. High. 2002. The extracytoplasmic sigma factor, final $\sigma^{\mathrm{E}}$, is required for intracellular survival of nontypeable Haemophilus influenzae in J774 macrophages. Infect. Immun. 70:708-715.

7. Datsenko, K. A., and B. L. Wanner. 2000. One-step inactivation of chromosomal genes in Escherichia coli K-12 using PCR products. Proc. Natl. Acad. Sci. USA 97:6640-6645.

8. De Las, P., L. Connolly, and C. A. Gross. 1997. $\sigma^{\mathrm{E}}$ is an essential sigma factor in Escherichia coli. J. Bacteriol. 179:6862-6864.

9. Elsinghorst, E. A. 1994. Measurement of invasion by gentamicin resistance. Methods Enzymol. 236:405-420.

10. Heungens, K., C. E. Cowles, and H. Goodrich-Blair. 2002. Identification of Xenorhabdus nematophila genes required for mutualistic colonization of Steinernema carpocapsae nematodes. Mol. Microbiol. 45:1337-1353.

11. Hild, E., K. Takayama, R. M. Olsson, and S. Kjelleberg. 2000. Evidence for a role of rpoE in stressed and unstressed cells of marine Vibrio angustum strain S14. J. Bacteriol. 182:6964-6974.

12. Hoiseth, S. K., and B. A. Stocker. 1981. Aromatic-dependent Salmonella typhimurium are non-virulent and effective as live vaccines. Nature 291:238239.

13. Humphreys, S., A. Stevenson, A. Bacon, A. B. Weinhardt, and M. Roberts. 1999. The alternative sigma factor, $\sigma^{\mathrm{E}}$, is critically important for the virulence of Salmonella typhimurium. Infect. Immun. 67:1560-1568.

14. Humphreys, S., G. Rowley, A. Stevenson, W. J. Kenyon, M. P. Spector, and M. Roberts. 2003. Role of periplasmic peptidylprolyl isomerases in Salmonella enterica serovar Typhimurium virulence. Infect. Immun. 71:5386-5388

15. Kanehara, K., K. Ito, and Y. Akiyama. 2002. YaeL (EcfE) activates the $\sigma^{\mathrm{E}}$ pathway of stress response through a site-2 cleavage of anti- $\sigma^{\mathrm{E}}$, RseA. Genes Dev. 16:2147-2155.

16. Kanehara, K., K. Ito, and Y. Akiyama. 2003. YaeL proteolysis of RseA is controlled by the PDZ domain of YaeL and a Gln-rich region of RseA. EMBO J. 22:6389-6398.

17. Kovacikova, G., and K. Skorupski. 2002. The alternative sigma factor $\sigma^{\mathrm{E}}$ plays an important role in intestinal survival and virulence in Vibrio cholerae. Infect. Immun. 70:5355-5362.

18. Linn, T., and R. St Pierre. 1990. Improved vector system for constructing transcriptional fusions that ensures independent translation of lacZ. J. Bacteriol. 172:1077-1084.

19. Miticka, H., G. Rowley, B. Rezuchova, D. Homerova, S. Humphreys, J. Farn, M. Roberts, and J. Kormanec. 2003. Transcriptional analysis of the $r p o E$ gene encoding extracytoplasmic stress response sigma factor $\sigma^{\mathrm{E}}$ in Salmonella enterica serovar Typhimurium. FEMS Microbiol. Lett. 226:307-314.

20. Redford, P., P. L. Roesch, and R. A. Welch. 2003. DegS is necessary for virulence and is among extraintestinal Escherichia coli genes induced in murine peritonitis. Infect. Immun. 71:3088-3096.

21. Richards, H., N. Datta, W. J. Sojka, and C. Wray. 1978. Trimethoprimresistance plasmids and transposons in Salmonella. Lancet 2:1194-1195.

22. Sambrook, J., E. F. Fritsch, and T. Maniatis. 1989. Molecular cloning: a laboratory manual, 2nd ed. Cold Spring Harbor Laboratory Press, Cold Spring Harbor, N.Y.

23. Slauch, J. M., and T. J. Silhavy. 1991. Genetic fusions as experimental tools. Methods Enzymol. 204:213-248.

24. Sydenham, M., G. Douce, F. Bowe, S. Ahmed, S. Chatfield, and G. Dougan. 2000. Salmonella enterica serovar Typhimurium surA mutants are attenuated and effective live oral vaccines. Infect. Immun. 68:1109-1115.

25. Testerman, T. L., A. Vazquez-Torres, Y. Xu, J. Jones-Carson, S. J. Libby, and F. C. Fang. 2002. The alternative sigma factor $\sigma^{\mathrm{E}}$ controls antioxidant defences required for Salmonella virulence and stationary-phase survival. Mol. Microbiol. 43:771-782.

26. Walsh, N. P., B. M. Alba, B. Bose, C. A. Gross, and R. T. Sauer. 2003. OMP peptide signals initiate the envelope-stress response by activating DegS protease via relief of inhibition mediated by its PDZ domain. Cell 113:61-71.

27. Yu, H., J. C. Boucher, N. S. Hibler, and V. Deretic. 1996. Virulence properties of Pseudomonas aeruginosa lacking the extreme-stress sigma factor $\mathrm{A} \lg \mathrm{U}\left(\sigma^{\mathrm{E}}\right)$. Infect. Immun. 64:2774-2781. 